\title{
Status of the muonic hydrogen Lamb-shift experiment ${ }^{1}$
}

\author{
T. Nebel, F.D. Amaro, A Antognini, F. Biraben, J.M.R. Cardoso, \\ C.A.N. Conde, A Dax, S. Dhawan, LM.P. Fernandes, \\ A Giesen, TW. Hansch, P. Indelicato, L. Julien, P.E Knowles, \\ F. Kottmann, E. Le Bigot, Y.-W. Liu, J.A.M. Lopes, L. Ludhova, \\ C.M.B. Monteiro, F. Mulhauser, F. Nez, R. Pohl, P. Rabinowitz, \\ J.M.F. dos Santos, LA. Schaller, K. Schuhmann, C. Schwob, \\ D. Taqqu, and J.F.C.A. Veloso
}

\begin{abstract}
The Lamb-shift experiment in muonic hydrogen $\left(\mu^{-} \mathrm{p}\right)$ aims to measure the energy difference between the $2 S_{1 / 2}^{F=1}-2 P_{3 / 2}^{F=2}$ atomic levels to a precision of $30 \mathrm{ppm}$. This would allow the r.m.s. proton charge radius $r_{\mathrm{p}}$ to be deduced to a precision of $10^{-3}$ and open a way to check bound-state quantum electrodynamics (QED) to a level of $10^{-7}$. The poor knowledge of the proton charge radius restricts tests of bound-state QED to the precision level of about $6 \times 10^{-6}$, although the experimental data themselves (Lamb-shift in hydrogen) have reached a precision of $2 \times 10^{-6}$. Values for $r_{\mathrm{p}}$ not depending on bound-state QED results from electron scattering experiments have a surprisingly large uncertainty of $2 \%$. In our Lamb-shift experiment, low-energy negative muons are stopped in low-density hydrogen gas, where, following the $\mu^{-}$atomic capture and cascade, $1 \%$ of the muonic hydrogen atoms form the metastable $2 S$ state with a lifetime of about $1 \mu \mathrm{s}$. A laser pulse at $\lambda \approx 6 \mu \mathrm{m}$ is used to drive the $2 S \rightarrow 2 \mathrm{P}$ transition. Following the laser excitation, we observe the $1.9 \mathrm{keV}$ $\mathrm{X}$-ray being emitted during the subsequent de-excitation to the $1 \mathrm{~S}$ state using large-area
\end{abstract}

T. Nebel, ${ }^{2}$ A. Antognini, T.W. Hansch, and R. Pohl. Max-Planck-Institutfur Quantenoptik, 85748 Garching, Germany.

F.D. Amaro, J.M.R. Cardoso, C.A.N. Conde, L.M.P. Fernandes, J.A.M. Lopes, C.M.B. Monteiro, J.M.F. dos Santos, and J.F.C.A. Veloso. Departamento de Fisica, Universidade de Coimbra, 3000 Coimbra, Portugal.

F. Biraben, P. Indelicato, L. Julien, E. Le Bigot, F. Nez, and C. Schwob. Laboratoire Kastler Brossel, ENS, UPMC, CNRS, 4 place Jussieu, 75252 Paris Cedex 05, France.

A. Dax and S. Dhawan. Physics Department, Yale University, New Haven, CT 06520-8121, USA.

A. Giesen and K. Schuhmann. Institut fur Strahlwerkzeuge, Universität Stuttgart, 70569 Stuttgart, Germany. P.E. Knowles, L. Ludhova,F. Mulhauser, and L.A. Schaller. Département de Physiquede l'Université, 1700

Fribourg, Switzerland.

F. Kottmann. Institut fur Teilchenphysik, ETH Zurich, 8093 Zurich, Switzerland.

Y.-W. Liu. Physics Department, National Tsing Hua University, Hsinchu 300, Taiwan.

P. Rabinowitz. Department of Chemistry, Princeton University, Princeton, NJ 08544-1009, USA.

D. Taqqu. Paul Scherrer Institute, 5232 Villigen-PSI, Switzerland.

'Paper given at the International Workshop on Precision Physics of Simple Atomic Systems, Venice International University, 12-16 June 2006.

'Corresponding author (e-mail: tbn@mpq.mpg.de). 
avalanche photodiodes. The resonance frequency and, hence, the Lamb shift and the proton charge radius are determined by measuring the intensity of the X-ray fluorescence as a function of the laser wavelength. The results of the run in December 2003 were negative but, nevertheless, promising. One by-product of the 2003 run was the first observation of the short-lived $2 S$ component in muonic hydrogen. Currently, improvements in the laser-system, the experimental apparatus, and the data acquisition are being implemented.

PACS Nos.: 36.10.Dr, 14.20.Dh, 42.62.Fi

Résumé : Notre expérience a pour but de mesurer le dkplacement de Lamb de l'hydrogkne muonique $\left(\mu^{-}\right.$p) plus précisément la diffkrence d'énergie entre les niveaux $2 S_{1 / 2}^{F=1}-2 P_{3 / 2}^{F=2}$ avec une incertitude relative de $30 \mathrm{ppm}$. Ceci permettrait de déterminer le rayon r.m.s. de charge du proton $r_{\mathrm{p}}$ avec une incertitude relative de $10^{-3}$ et par la suite de tester les calculs d'électrodynamiques quantiques sur des états liés avec une incertitude relative de $10^{-7}$. L'incertitude actuelle sur $r_{\mathrm{p}}$ limite la précision des calculs QED des états liks à $6 \times 10^{-6}$ alors que les données expérimentales (dkplacement de Lamb dans l'hydrogkne) sont connues avec une incertitude relative de $2 \times 10^{-6}$. Les dkterminations de $r_{\mathrm{p}}$ indkpendantes des calculs QED d'états liks provient d'expérience de diffusion d'électrons et ont une incertitude relative, ktonnamment grande, de $2 \%$. Dans notre expkrience, des muons nkgatifs de basse énergie sont arrêtés dans un gaz d'hydrogkne de faible densité ou après la capture des muons négatifs puis la cascade radiative, $1 \%$ des atomes d'hydrogkne muonique sont formés dans l'ktat mktastable $2 S$ de durée de vie $1 \mu$ s environ. Une impulsion laser $(\lambda=6 \mathrm{pm})$ induit la transition $2 S \rightarrow 2 \mathrm{P}$. Après l'excitation laser, nous observons grâce à des photodiodes à avalanches de grande surface les rayons $\mathrm{X}$ à $1,9 \mathrm{keV}$ émits par durant la désexcitation vers l'ktat $1 S$. La fréquence de cette rksonance et donc le dkplacement de Lamb et le rayon du proton sont déterminés en mesurant l'intensitk de la fluorescence des rayons $\mathrm{X}$ en fonction de la longueur d'onde laser. Les rksultats des enregistrements faits en décembre 2003 sont nkgatifs mais pourtant prometteurs. Ainsi, durant les enregistrements 2003, la composante de dksexcitation rapide de l'ktat $2 S$ a été observée pour la première fois dans l'hydrogkne muonique. Actuellement, nous amkliorons le systkme laser, le dispositif expérimental et le systkme d'acquisition des données.

\section{I- Introduction}

Values for the r.m.s. charge radius $r_{\mathrm{p}}$ of the proton, the simplest nucleus that does not depend on bound-state quantum electrodynamics (QED), are known only with a surprisingly low precision of about $2 \%$. Recent re-evaluation of all the electron scattering data [1] yields a value of $0.897(18) \mathrm{fm}$. The poor knowledge of the proton charge radius restricts tests of bound-state QED to the precision level of about $6 \times 10^{-6}$, although data from Lamb-shift experiments in hydrogen have reached a precision of $2 \times 10^{-6}$. The determination of the proton charge radius with an accuracy of $10^{-3}$ is the main goal of our collaboration.

We aim to measure the $2 S$ Lamb shift in muonic hydrogen $\left(\mu^{-} \mathrm{p}\right)$. Vacuum polarization dominates the $\mu^{-} \mathrm{p}$ Lamb shift, $\Delta E_{2 P-2 S} \approx 0.2 \mathrm{eV}$, and alters the $2 S$ binding energy towards more negative values. The fine and hyperfine structure splittings in muonic hydrogen are an order of magnitude smaller than the Lamb shift. The key motivation for our experiment is found in the nucleus' finite-size contribution to the $\mu^{-} \mathrm{p}$ Lamb shift, originating from a large overlap of the muon's and proton's wave functions $\left(\mathrm{m}, \approx 207 \mathrm{~m}_{\mathrm{e}}\right.$ ). This effect is as high as $1.8 \%$, two orders of magnitude larger than in ordinary hydrogen. Combining the $\mu^{-} \mathrm{p}$ Lamb-shift measurement with the precision of hydrogen spectroscopy will ultimately lead to a test of bound-state QED approaching the level of $10^{-7}[2,3]$.

The Lamb-shift experiment is performed at the muon beam line at the Paul Scherrer Institute (PSI) in Switzerland [4]. By means of laser spectroscopy ( $\lambda \approx 6 \mu \mathrm{m}, v \approx 50 \mathrm{THz}$ ), we want to measure the energy difference $\triangle E\left(2 S_{1 / 2}^{F=1}-2 P_{3 / 2}^{F}=2\right)$ to a precision of $30 \mathrm{ppm}$. This corresponds to $10 \%$ of the natural line width (given by the $2 \mathrm{P}$ lifetime). From there, one can deduce the r.m.s. proton charge radius 
$r_{\mathrm{p}}$ with $10^{-3}$ relative accuracy, 20 times more accurate than presently known.

The basic idea of the experiment is to stop negative muons in low-pressure $(0.6 \mathrm{hPa})$ hydrogen $\left(\mathrm{H}_{2}\right)$ gas where $\mu^{-} \mathrm{p}$ atoms in highly excited states $(\mathrm{n} \approx 14)$ are formed [5]. Through a series of different de-excitation processes (see, for example, refs. 5 and 6 ), most of the $\mu^{-} \mathrm{p}$ atoms quickly cascade down to the $1 S$ ground state. Only $\sim 1 \%$ form long-lived $\mu^{-}$p atoms in the $2 S$ state $[7,8]$. About $1 \mu$ s after the muon cascade, a short laser pulse with a tunability of $250 \mathrm{GHz}$ illuminates the muon stop volume. When the laser is on resonance, the $\mu^{-} \mathrm{p}$ atoms are excited to the $2 P$ state from where they decay to the $1 \mathrm{~S}$ ground state immediately (lifetime $\tau_{2 P}=8.6 \mathrm{ps}$ ). The emitted $1.9 \mathrm{keV} \mathrm{X-rays} \mathrm{serve} \mathrm{as} \mathrm{a} \mathrm{signal}$ and are recorded versus the laser wavelength.

Several phenomena affect the lifetime of the $2 S$ state significantly and, therefore, put stringent constraints on our setup, in particular, on the laser system. The dominant quenching channel is the resonant formation of an excited muonic molecule $[8,9]$

$\mu \mathrm{p}(2 S)+\mathrm{H}_{2} \rightarrow\left\{\left[(\mathrm{pp} \mu)^{+}\right]^{*} \mathrm{pee}\right\}^{*} \rightarrow \mu \mathrm{p}(1 S)+\mathrm{p}+\cdots+1.9 \mathrm{keV}$

With the laser used during the run in 2003, the fastest laser response time achieved, i.e., the delay time between the detected stopped muon and the laser pulse, was $-1.5 \mu$ s. Therefore, the working $\mathrm{H}_{2}$ target gas pressure had to be as low as $0.6 \mathrm{hPa}$ (at room temperature) to reduce collisional quenching and to ensure a reasonable $2 S$ lifetime of about $1.3 \mu \mathrm{s}$ [7]. Such a low gas pressure was one of the main challenges during the experiment.

In the following section, the experimental setup, including the muon beam and the gas target, will be introduced. Section 3 deals with the laser system used during the 2003 run, its limitations, aid the improvements currently being implemented. Although no resonance line was found during the last experimental run in 2003, interesting results were obtained. They are presented in Sect. 4. In Sect. 5, means to improve the event rate are discussed.

\section{Experimental setup}

The muonic hydrogen Lamb-shift experiment consists of the muon beam line, the target cavity, the $\mathrm{X}$-ray detectors, and the laser system. Collisional quenching of the $2 S$ state made it necessary to stop the negative muons in low-pressure hydrogen gas at $0.6 \mathrm{hPa}$. To stop muons in gas of such low density, their kinetic energy has to be less than $5 \mathrm{keV}$. A novel low-energy muon beam was, therefore, developed at PSI to efficiently decelerate muons produced at MeV energies within the muon lifetime of $2.2 \mu \mathrm{s}$.

The beam line consists of the cyclotron trap (CT) for the production of low-energy muons [10], the muon extraction channel (MEC) [4] to transport and select the muons, and two transmission muon detectors as well as the gas target, both enclosed in a $5 \mathrm{~T}$ solenoid. Negative pions $\left(10^{8} \mathrm{~s}^{-1}\right)$ with a momentum of $102 \mathrm{MeV} / \mathrm{c}$ are injected tangentially into the median plane of the cyclotron trap aid moderated by passing a degrader. The CT consists of a pair of superconducting ring coils (spaced $40 \mathrm{~cm}$ apart) that form a magnetic trap with a magnetic field of $4 \mathrm{~T}$ at the coil centers and $2 \mathrm{~T}$ in the medial plane. About $30 \%$ of the decelerated pions decay into muons in flight, and a few percent of these have suitable momenta to be stored in the magnetic bottle. The confined muons are further moderated by repeatedly crossing a $200 \mathrm{~nm}$ thin Formvar ${ }^{\circledR}$ foil (sputtered with a $3 \mathrm{~nm}$ thick nickel layer to make it conductive) located at the center of the trap until they reach energies of $10-50 \mathrm{keV}$. At sufficiently low muon energies, the repulsive electric field applied to the moderator foil dominates over the magnetic confinement, and muons leave the CT axially. They then enter the muon extraction channel, acting as a momentum filter, which is made up from a toroidal assembly of magnetic coils (magnetic field of $0.15 \mathrm{~T}$ ). It has a high transmission for muons of $20 \mathrm{keV}$ energy and separates the beam from unwanted particles like high-energy muons and electrons, as well as neutrons and gamma rays.

Then the $\mu^{-}$enter a $5 \mathrm{~T}$ solenoid, whose magnetic field keeps the axial dimensions of the muon beam as small as possible. This ensures that the target volume has a minimal radial size to maximize 
the laser-induced transition probability and the solid-angle for the X-ray detection. Before entering the hydrogen target, the muons pass two transmission detectors where they are decelerated to $3-6 \mathrm{keV}$ and their phase space is compressed slightly [11]. Two stacks of ultra-thin carbon foils $\left(d=4 \mu \mathrm{g} / \mathrm{cm}^{2}\right)$ kept at high voltage release a few electrons when a muon is crossing the foils [12]. These electrons are accelerated in the electricfield of the stacks and then separated spatially by an $\boldsymbol{E} \times \boldsymbol{B}$ separator field from the much slower muons. The electrons are finally detected by plastic scintillators and provide the trigger signal for the laser system and the data acquisition. Combined with an anti-coincidence-detector, the transmission detector efficiency is $37 \%$. The entire beam line delivers around 240 detected and usable muons per second.

Having passed the transmission detectors, the muons enter the gas target volume [12] through a $30 \mathrm{~nm}$ thin Formvar ${ }^{\circledR}$ entrance window. The target volume is filled with $0.6 \mathrm{hPa}$ of $\mathrm{H}_{2}$ gas and has a length of $20 \mathrm{~cm}$ along the beam direction. This length is dictated by the stop distribution of the muons in such low-pressure $\mathrm{H}_{2}$ gas. The pencil-shaped muon stop volume is surrounded by two cylindrical high-reflectivemirrors, which form a nonresonant multipass cavity, providing an almost homogenous irradiation by the $6 \mu \mathrm{m}$ laser light. An energy density of $16.5 \mathrm{~mJ} / \mathrm{cm}^{2}$ is needed to saturate the $2 S-2 P$ transition [13]. Twenty large-area avalanche photodiodes (LAAPD) are mounted above and below the muon stop volume and record the $1.9 \mathrm{keV} 2 \mathrm{P}-1 \mathrm{~S} \mathrm{X}$-rays [14] in two face-to-face rows of 10 devices at a vertical distance of only $8 \mathrm{~mm}$ from the muon beam center. They are cooled down to $-30^{\circ} \mathrm{C}$ to achieve an optimal energy resolution and signal-to-noise ratio.

\section{Laser system}

The laser system used to produce the $6 \mu \mathrm{m}$ radiation is described in this section. Only the muon beam at PSI can deliver a sufficient rate of low-energy $\mu^{-}$. This beam, however, is not pulsed, which puts tight constraints on our laser system. To have an event rate as high as possible, the laser has to be triggered by the muons entering the muon stop volume at stochastic times, with a delay time smaller than about $1 \mu \mathrm{s}$ (because of the lifetime of $1.3 \mu \mathrm{s}$ of the $2 S$ state at the working pressure), and with the shortest possible dead time. Furthermore, the scanning range of the laser has to be from 6.00 to $6.03 \mu \mathrm{m}$ due to the uncertainty in the proton radius. To reach the needed energy density of $16.5 \mathrm{~mJ} / \mathrm{cm}^{2}$ inside the target cavity, the $6 \mu \mathrm{m}$ laser pulses have to have an energy of $\approx 0.2 \mathrm{~mJ}$ at the cavity entrance. The laser bandwidth is required to be small compared to the natural linewidth of $18 \mathrm{GHz}$ of the $2 S-2 P$ transition, i.e., of the order of $1 \mathrm{GHz}$. Having a reliable system during the beam-time of several weeks is crucial.

The $6 \mu \mathrm{m}$ light pulses $(0.2 \mathrm{~mJ})$ enter the enhancement cavity through a tiny hole $(d=0.63 \mathrm{~mm})$ in one of the mirror substrates. As shown in Fig. 1 (lowerpart), the $6 \mu$ m light is convertedfrom $708 \mathrm{~nm}$ light pulses using an efficient and reliable 3rd-Stokes-Raman shifter operated with $14 \mathrm{bar}_{2}$ gas [15]. The red $708 \mathrm{~nm}$ pulses $\left(\mathrm{TEM}_{00}\right.$ mode, width $6 \mathrm{~ns}$ FWHM, energy $12 \mathrm{~mJ}$ ) needed for the Raman-scattering process are provided by a titanium sapphire (Ti:Sa) oscillator-amplifier system. The Ti:Sa oscillator consists of a $7 \mathrm{~cm}$ short wavelength-selectivecavity and emits $1.2 \mathrm{~mJ}$ pulses at $708 \mathrm{~nm}$. The wavelength tunability and bandwidth of the Ti:Sa oscillator, and, therefore, of the subsequent amplifier and $6 \mu \mathrm{m}$ light, are controlled by a single-mode cw-Ti:Sa laser stabilized on an external calibrated Fabry-Perot (FP) cavity. The measured frequency chirp in the oscillator is about $100 \mathrm{MHz}$ and is confirmed by theoretical models.

Both, the Ti:Sa oscillator and multipass amplifier are pumped with green pulses of around $40 \mathrm{~mJ}$ energy. During the last measuring run in 2003, the whole laser system was pumped by two high-power $\mathrm{XeCl}$ excimer lasers delivering an energy per pulse of $350 \mathrm{~mJ}$ each. Each excirner laser was pumping a two-stage multimode dye laser that converted the $308 \mathrm{~nm}$ excimer laser pulses to green light pulses at $540 \mathrm{~nm}$. It turned out that the $1.2 \mu \mathrm{s}$ delay time of the excimer lasers between the electrical trigger and the light pulse was too large. Since the laser was stochastically triggered, the dead time between two consecutive pulses had to be set to $14 \mathrm{~ms}$ to avoid energy fluctuations. With a muon trigger rate of 
Fig. 1. Schematic view of the new laser system to be used in 2007. The main components are a pulsed thin-disc laser system, a tunable cw Ti:Sa laser, a pulsed oscillator-amplifier Ti:Sa laser, a Raman cell, and a $6 \mu \mathrm{m}$ mirror cavity with its diagnostic system. FP: Fabry-Perot, $\mathrm{I}_{2}$ : iodine absorption cell, $\mathrm{H}_{2} \mathrm{O}$ : water absorption cell, PZT: piezo transducer, FI: Faraday Isolator.

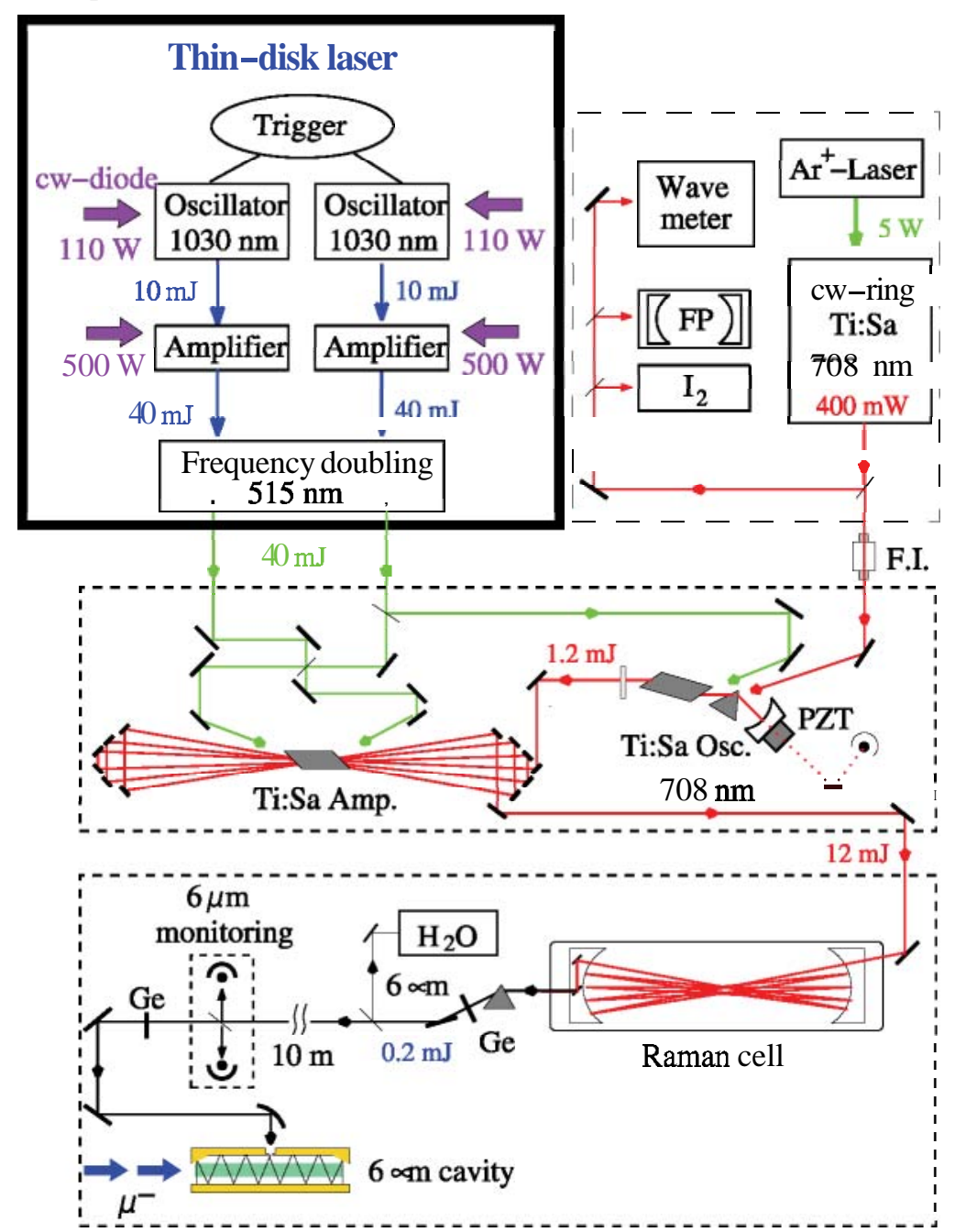

$240 \mathrm{~s}^{-1}$ this resulted in an average laser repetition rate of $55 \mathrm{~s}^{-1} \simeq(1 / 240+0.014)^{-1} \mathrm{~s}^{-1}$. Therefore, the old pumping stage is currently replaced by a new thin-disc laser. Figure 1 shows a scheme of the whole laser system including the new thin-disc laser.

Thin-disc lasers [16] avoid the problem of thermal lensing and destroying the laser crystal by using a very thin $(200 \mu \mathrm{m})$ gain medium (Ytterbium-YAG), which is cooled axially to obtain a flat-top refractive index profile (Fig. 2a). The disc-laser pumping unit is set up as a master oscillator, power amplifier(MOPA) device. While the amplifier follows a common multipassconfiguration adapted to the disc-laser geometry, the oscillator is Q-switched using a Pockels cell (Fig. 2b). It delivers $10 \mathrm{~mJ}$ pulses at $1030 \mathrm{~nm}$ at a repetition rate of $400 \mathrm{~Hz}$ with a beam-quality factor $M^{2}=1.2$ and an internal delay of only $300 \mathrm{~ns}$. Such a short delay is achieved by operating the oscillator in a cw-prelasing mode. When triggered, the cavity is closed, causing a fast pulse build-up. After a short time, the cavity is opened 
Fig. 2. Principle of the new thin-disc laser developed for the Lamb-shift experiment. (a) The thin laser crystal has a highly reflective coating at the back side, which is soldered onto a copper heat-sink. It is cooled axially from the back to avoid thermal lensing and acts as a mirror for the laser cavity. The pump beam is redirected onto the crystal several times using folding mirrors (not shown) to absorb enough pump power. (b) In the oscillator, the Pockels cell (PC) and the quarter-wave plate rotate the polarization such that the thin film polarizer (TFP) (slightly) reflects or transmits the circulating light to form a prelasing, a build-up, or an open cavity, respectively.

(a)

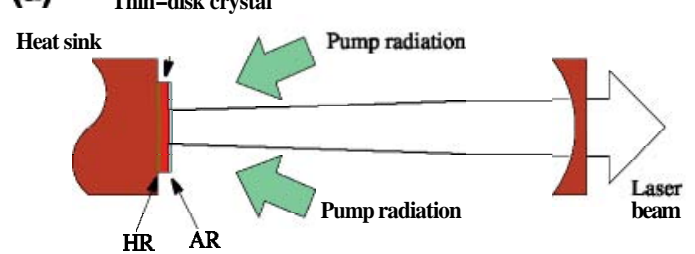

(b)

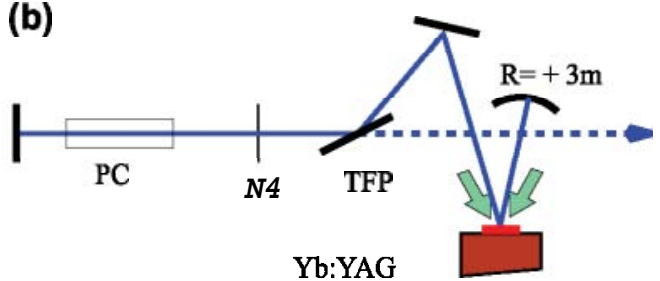

Fig. 3. Energy and time spectrum of the recorded data. (a) The X-ray energy spectrum (shown here from one LAAPD). The continuous lines represent fits of the $\mathrm{K}_{\alpha}$, the $\mathrm{K}_{\beta}$, and the $\mathrm{K}_{\text {rest }}$ contributions (plus a weak background line at $3.1 \mathrm{keV}$ ). (b) Time spectrum of $2 \mathrm{keV}$ X-rays. The laser time window is indicated.
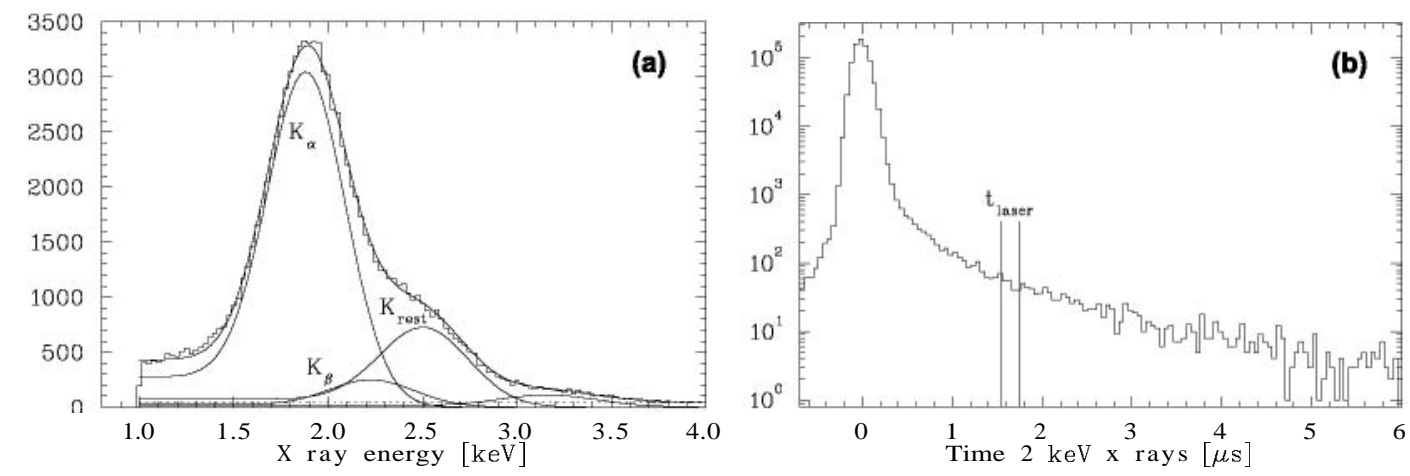

completely and the circulating power is released. A set of two such systems is pumped with a total of about $1200 \mathrm{~W}$ of cw-laser light at $940 \mathrm{~nm}$; the emitted light $(1030 \mathrm{~nm})$ is frequency doubled to $515 \mathrm{~nm}$ before pumping the Ti:Sa system.

\section{Results from the 2003 run}

Using the experimental setup including the mentioned excimer-dye pumping laser stage (described in more detail in ref. 12), the data presented in this section were obtained. During three weeks of measurement at PSI, more than $10^{6} \mathrm{X}$-rays from $\mu^{-} \mathrm{p}$ K-Line transitions $\left(\mathrm{K}_{\alpha}, \mathrm{Kg}\right.$, and $\mathrm{K}_{\text {rest }}$, at 1.895 , 2.246 , and $2.44 \mathrm{keV}$, respectively) were recorded at $0.6 \mathrm{hPa} \mathrm{H}_{2}(\mathrm{~T}=290 \mathrm{~K})$. Due to its dead time, the laser was fired for only $\approx 30 \%$ of the detected muons. To determine the background with optimum statistics, additional events were recorded between laser shots. For one LAAPD, the X-ray energy spectrum of all recorded X-raysis shown in Fig. 3a. The relative intensitiesof the $\mu^{-} \mathrm{p} \mathrm{K}$-lines, corrected for the energy-dependent detector efficiency, correspond to previous measurements [17].

The time spectrum of all good data taken (Fig. $3 \mathrm{~b}$ reveals a prompt peak at $t=0$, which originates from the majority of the $\mu^{-} \mathrm{p}$ atoms that do not form metastable $\mu^{-} \mathrm{p}(2 S)$ atoms. Instead, they de-excite to the $\mu^{-} \mathrm{p}(1 S)$ ground state, thereby emitting a muonic K-line X-ray. The delayed background seen in Fig. 3b) can be mainly attributed to muonic carbon X-rays: $\mu^{-} \mathrm{p}(1 S)$ atoms hitting the target walls transfering the muon to the carbon resulting in 5 and $14 \mathrm{keV} \mathrm{X-rays} \mathrm{that} \mathrm{can} \mathrm{show} \mathrm{up} \mathrm{as} \mathrm{small} \mathrm{signals} \mathrm{in}$ 
Fig. 4. Results of the $2 P-2 S$ resonance search in 2003. The dots represent the number of measured events in a $200 \mathrm{~ns}$ wide laser time window. The Fabry-Perot (FP) fringe number is a measure of the laser frequency. The scanned region (49.7409-49.8757 THz) corresponds to a proton charge radius of 0.844$0.905 \mathrm{fm}$. The confidence levels are calculated for the hypothesis that only background is present. The upper plot shows the approximate measuring time per point.

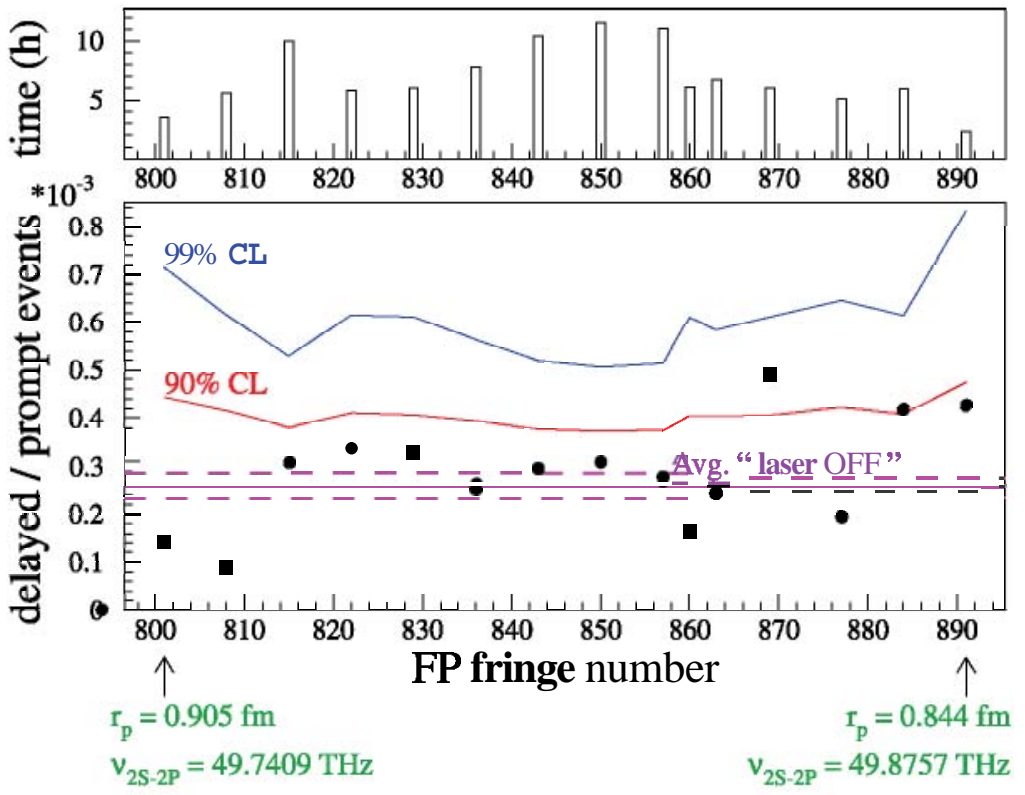

the $2 \mathrm{keV}$ region when measured by the LAAPDs. The events of interest are located in a time window of $200 \mathrm{~ns}$ after the laser has illuminated the target volume. The relative energy resolution of the LAAPDs is around $25 \%$ and the time resolution $35 \mathrm{~ns}$ for $2 \mathrm{keV}$ X-rays [14].

The final result of the 2003 run is shown in Fig. 4. For each wavelength (shown in units of the FP fringe number), we plotted the number of delayed events in the laser time window divided by the number of prompt events, thereby normalizing to the true number of useful laser shots at this wavelength. The expected number of backgroundevents is shown as the "avg. laser OFF" line, and the lines "90\% CL" and "99\% CL" indicate the 90 and 99\% confidencelevels for the hypothesis"data contain only background".

No resonance line was discovered during the 2003 run. Nevertheless, the statistics are too low to exclude the r.m.s. proton charge radius being in the interval of $0.844-0.905 \mathrm{fm}$. The main sources of background were analyzed and are depicted in Fig. 5 [13]. It shows the measured 2 keV-background $\mathrm{X}$-ray time spectrum. Its fit function contains four components. The first component (1) comes from muon transfer to carbon. This can happen when a $\mu^{-} \mathrm{p}$ atom hits the polypropylene foils installed in front of the LAAPDs. The muon is then transferred to form $\left(\mu^{-} \mathrm{C}\right)^{*}$ which de-excites emitting X-rays of $4.9 \mathrm{keV}$ energy. These can show up as $2 \mathrm{keV}$ background. Another component (2) comes from falsely identified muon-decay electrons imitating a laser-induced good event.

When a muon was detected, it triggered the laser and the data acquisition, and opened an event gate in which X-ray events were recorded. During the open event gate, a second muon could enter the target volume at random times, form a $\mu^{-}$p atom emitting a $2 \mathrm{keV} \mathrm{X-ray,} \mathrm{and} \mathrm{be} \mathrm{misinterpreted} \mathrm{as} \mathrm{an} \mathrm{X-ray}$ event belonging to the first muon. This process caused the flat background component (3). The fourth component (4) of $2 \mathrm{keV} X$-ray events comes from $\mu^{-} \mathrm{p}(2 S)$ atoms, which have enough kinetic energy after the muonic cascade to be radiatively quenched to the ground state, thereby emitting a $2 \mathrm{keV} \mathrm{X-ray.}$ 
Fig. 5. Measured 2 keV-background X-ray spectrum. The bin width is $200 \mathrm{~ns}$. The ordinate gives the measuring probability per formed $\mu^{-} \mathrm{p}$ atom. The total fit function is a sum of four components: (1) the background component related to the muon transfer to carbon; (2) the background related to falsely identified muon-decay electrons; (3) a flat background component caused by undetected second muons; and (4) events coming from the radiative de-excitation of the short-lived $2 S$ component of $\mu^{-} \mathrm{p}$. The two vertical lines define the laser time window.

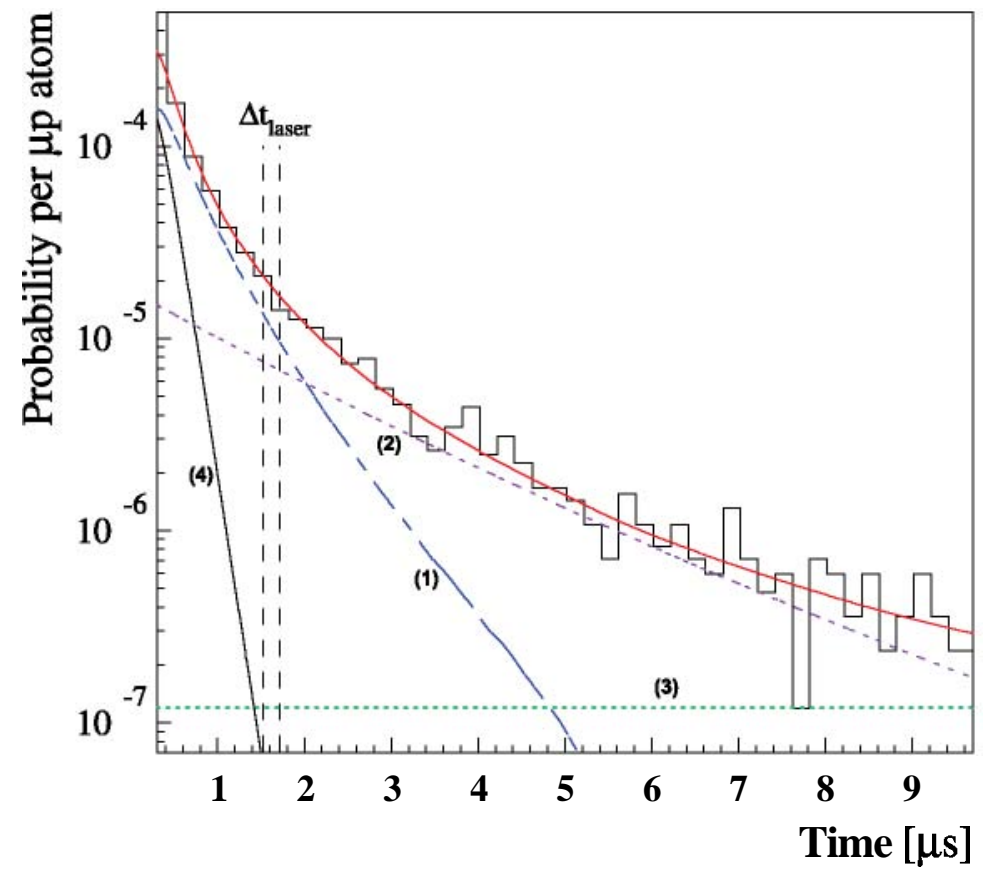

Although no resonanceline was found during the run in 2003, the contributions to the background were well analyzed to be able to be addressed for future improvements.

\section{Conclusion}

During the 2003 run, the signal-to-background ratio was of the order of one, and the event rate was only $0.5 \mathrm{~h}^{-1}$. For these reasons, a clearly distinguishable resonance line could not be discovered. This can be seen in Fig. 6, a plot of 16 Monte Carlo simulated examples with the parameters found during the 2003 experiment. In more than half of the spectra, the resonance line is well hidden below the background. Several measures are being taken while preparing for the 2007 run to detect a resonance line. As mentioned in Sect. 3, a considerable improvement stems from the shorter delay of the laser system, reduced from $1.2 \mu$ s to $300 \mathrm{~ns}$.

Furthermore, in 2003, the LAAPDs had to be shielded by thin Li-foils from a-particles emitted from a radioactive targetcavity coating. These foils absorbed the a-particles but also $50 \%$ of the signal X-rays. A new nonradioactive coating will make the Li-foils obsolete. In addition, avoiding the muon transfer to carbon in the polypropylenefoils will reduce the background significantly. This will be achieved by placing a thin gold layer onto the polypropylenefoils, producing $\left(\mu^{-} \mathrm{Au}\right)^{*}$ instead of $\left(\mu^{-} \mathrm{C}\right)^{*}$. Because of the strongly reduced muon lifetime in gold ( $\tau_{\mu}^{\mathrm{Au}}=73 \mathrm{~ns}$ ) [18], the X-rays and Auger electrons emitted during the $\mu^{-}$Au cascade do not show up as signal events in the laser time window. Figure 7 shows the very promising result of a Monte Carlo simulation with the expected parameters for the run in 2007. 
Fig. 6. Monte Carlo simulation of the resonance search in 2003. Using values corresponding to the parameters of 2003, a signal rate of 0.5 events per hour, a signal-to-background ratio of $\mathbf{1}$, and a measuring time of $7 \mathrm{~h}$ at each data point were assumed. The resonance in this simulation is centered on zero frequency. More than half of the spectra do not show a clear resonance effect.

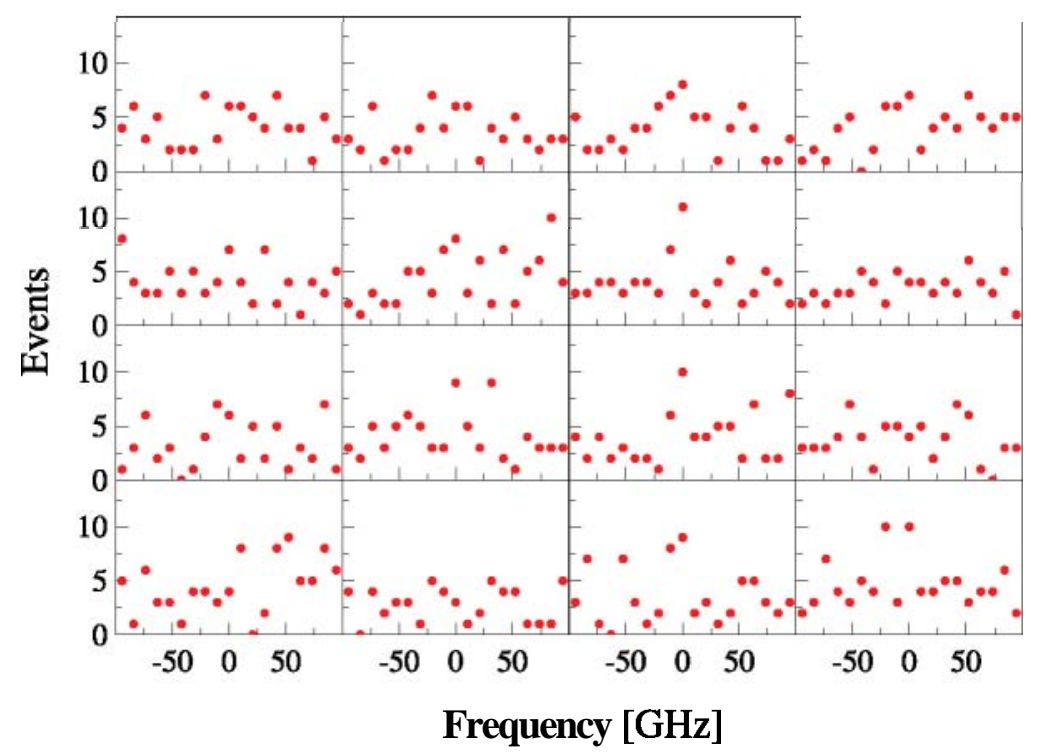

Fig. 7. Simulated examples of the prospective $2 S-2 P$ resonance search in 2007 . Using values expected from the current improvements, a signal rate of 10 events per hour, a signal-to-background ratio of 3 , and a measuring time of $2 \mathrm{~h}$ at each data point were assumed. In all spectra, a resonance centered on zero frequency is clearly visible.

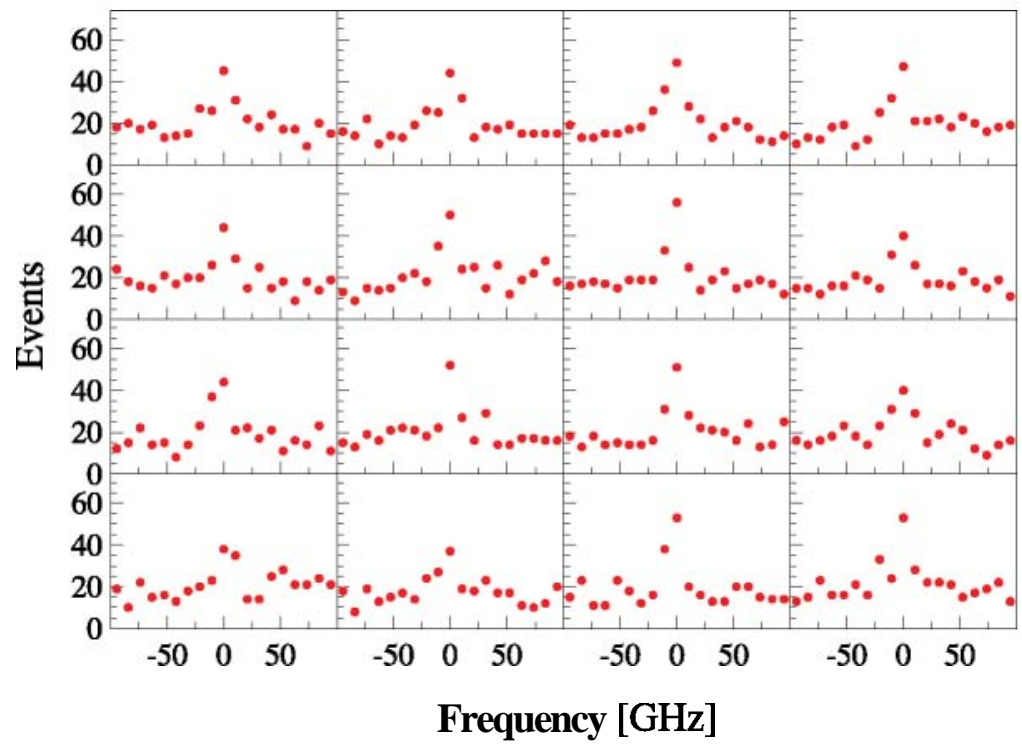

\section{Acknowledgments}

This work was performed at the Paul Scherrer Institute, Switzerland. We acknowledge support from the Swiss National Science Foundation, the Swiss Academy of Engineering Sciences, BQR de 1'UFR 
de physique fondamentale et appliquée de l'Université Paris 6, the program PAI Germaine de Stael $\mathrm{n}^{\circ} 07819 \mathrm{NH}$ du ministère des affaires étrangères France, Portuguese Foundation for Science and Technology FCT and FEDER under project POCTI/FNU/41720/2001, and the US Department of Energy. The authors would like to thank L. Simons and B. Leoni for setting up the cyclotron trap; O. Huot and Z. Hochman for technical support; T. Gerber with his Reaction Analysis Group, and R. Bombach for providing lasercomponents and helpful advice; H.-J. Kluge and W. Nörthershäuser of GSIDarmstadtfor providing dye pumps, excimer, and Art lasers; R. Zenobi and A. Renn of the Department of Chemistry at Swiss Federal Institute of Technology in Zurich (Switzerland) for providing a dye pump and an $\mathrm{Ar}^{+}$

laser; and H. van Den Bergh of the Swiss Federal Institute of Technology in Lausanne (Switzerland) for an excimer laser. We also thank the PSI accelerator division, the Hallendienst, the workshops at PSI, MPQ, and Fribourg, and other support groups for their valuable help.

\section{References}

1. P.G. Blunden and I. Sick. Phys. Rev. C, 72, 057601 (2005).

2. B. de Beauvoir, C. Schwob, O. Acef, L. Jozefowski, L. Hilico, F. Nez, L. Julien, A. Clairon, and F. Biraben. Eur. Phys. J. D, 12, 61 (2000).

3. M. Niering, R. Holzwarth, J. Reichert, P. Pokasov, T. Udem, M. Weitz, T.W. Hansch, P. Lemonde, G. Santarelli, M. Abgrall, P. Laurent, C. Salomon, and A. Clairon. Phys. Rev. Lett. 84, 5496 (2000).

4. F. Kottmann, A. Antognini, F. Biraben et al. In Proceedings of the International Workshop on Exotic Atoms (EXA02). Edited by P. Kienle, J. Marton, and J. Zmeskal. Austrian Acad. of Sciences, Vienna. 2003. p. 159.

5. T.S. Jensen and V.E. Markushin. Eur. Phys. J. D, 21, 271 (2002).

6. L. Ludhova. Ph.D. thesis, University of Fribourg, Switzerland. 2005.

7. R. Pohl, H. Daniel, EJ. Hartmann et al. Hyperfine Int. 138, 35 (2001).

8. R. Pohl, H. Daniel, F. Hartmann, P. Hauser, F. Kottmann, V.E. Markushin, M. Miihlbauer, C. Petitjean, W. Schott, D. Taqqu, and P. Wojciechowski-Grosshauser. Phys. Rev. Lett. 97, 193402 (2006).

9. J. Wallenius, S. Jonsell, Y. Kino, and P. Froehlich. Hyperfine Int. 138, 285 (2001).

10. P de Cecco, P. Hauser, D. Horvàth, F. Kottmann, L.M. Simons, and D. Taqqu. Nucl. Instrum. Methods A, 394, 287 (1997).

11. M. Miihlbauer, H. Daniel, EJ. Hartmann, P. Hauser, F. Kottmann, C. Petitjean, W. Schott, D. Taqqu, and P. Wojciechowski. Hyperfine Int. 119, 305 (1999).

12. R. Pohl, A. Antognini, ED. Amaro, F. Biraben, J.M.R. Cardoso et al. Can. J. Phys. 83, 339 (2005).

13. A. Antognini. Ph.D. thesis, Ludwig Maximilians University, Munich, Germany. 2005.

14. L. Ludhova, ED. Amaro, A. Antognini, F Biraben, J.M.R. Cardoso, C.A.N. Conde, A. Dax, S. Dhawan, L.M.P. Fernandes, T.W. Hansch et al.. Nucl. Instrum. Methods A, 540, 169 (2005) and references therein.

15. P. Rabinowitz, B.N. Perry, and N. Levinos. IEEE J. Quantum. Elect. 22, 797 (1986).

16. A. Giesen, H. Hugel, A. Voss, K. Wittig, U. Brauch, and H. Opower. Appl. Phys. B, 58, 365 (1994).

17. H. Anderhub, H.P. Vonarb, J. Bocklin, F. Dittus, R.F. Marques, H. Hofer, F. Kottmann, D. Taqqu, and J. Unternahrer. Phys. Lett. 143B, 65 (1984).

18. T. Suzuki, D.F. Measday, and J.P. Roalsvig. Phys. Rev. C, 35, 2212 (1987). 